\title{
Clima social familiar y habilidades sociales en estudiantes de las instituciones educativas de San Juan de Lurigancho, Perú
}

\author{
Family social climate and social skills in students of the educational institutions of San Juan de Lurigancho, Peru \\ Clima social familiar e habilidades sociais em alunos das instituições de ensino de San Juan de Lurigancho, Peru
}

Zara Graciela Pablo Ricra

tex.zara13@gmail.com

https://orcid.org/0000-0001-9233-8866

Universidad César Vallejo, Lima-Perú

Giuliana del Socorro Raggio Ramírez

giulianita2706@gmail.com

https://orcid.org/0000-0002-0082-2050

Universidad César Vallejo, Lima-Perú
Isabel Menacho Vargas

isabelmenachov@gmail.com

https://orcid.org/0000-0001-6246-4618

Universidad César Vallejo, Lima-Perú

Gisella Socorro Flores Mejía

gisellaflores63@hotmail.com

https://orcid.org/0000-0002-1558-7022

Universidad César Vallejo, Lima-Perú

Artículo recibido en junio 2021, revisado en julio 2021, arbitrado en agosto 2021 y publicado en septiembre 2021

\section{RESUMEN}

El estudio tuvo como objetivo determinar la relación entre el clima social familiar en la dimensión relación y las habilidades sociales en estudiantes de las instituciones educativas de San Juan de Lurigancho, Perú. El tipo de investigación fue una investigación básica y el diseño fue no experimental, transversal-correlacional. Para llevar a cabo la investigación se empleó la técnica de la encuesta que evalúa las dos variables estudiadas y se utilizó como instrumento un cuestionario. Para contrastar las hipótesis, se empleó la estadística no paramétrica, Rho de Spearman. Los resultados obtenidos según las pruebas de hipótesis, para la hipótesis general, el valor de error calculado entre las variables, tiene como resultado $(3,4207 \mathrm{E}$ $63)$ el cual es inferior al margen $(0,05)$ por lo que se puede afirmar que existe correlación. Con respecto al coeficiente de correlación se consiguió $(0,944379)$ por lo que se comprueba que la correlación ha sido positiva y muy alta entre las habilidades sociales y las dimensiones del clima social familiar (relación, desarrollo y estabilidad), donde se obtuvo un valor de 0,$870704 ; 0,903853$ y 0,903853 respectivamente.

Palabras clave: Relación intrafamiliar; Procesos de aprendizaje social; Apoyo familiar

\section{ABSTRACT}

The objective of the study was to determine the relationship between the family social climate in the relationship dimension and the social skills in students of the educational institutions of San Juan de Lurigancho, Peru. The type of research was basic research and the design was non-experimental, cross-correlational. To carry out the research, the survey technique that evaluates the two variables studied was used and a questionnaire was used as an instrument. To test the hypotheses, the nonparametric statistic, Spearman's Rho, was used. The results obtained according to the hypothesis tests, for the general hypothesis, the error value calculated between the variables, has as a result (3.4207E-63) which is less than the margin $(0.05)$ so it can be stated that there is correlation. Regarding the correlation coefficient, (0.944379) was obtained, which is why it is verified that the correlation has been positive and very high between social skills and the dimensions of the family social climate (relationship, development and stability), where a value of $0.870704 ; 0.903853$ and 0.903853 respectively.

Key words: Intra-family relationship; Social learning processes, Family support

\section{RESUMO}

O objetivo do estudo foi determinar a relação entre o clima social familiar na dimensão relacional e as habilidades sociais em alunos das instituições de ensino de San Juan de Lurigancho, Perú. O tipo de pesquisa foi a pesquisa básica e o desenho não experimental, correlacional cruzado. Para a realização da pesquisa, foi utilizada a técnica de levantamento que avalia as duas variáveis estudadas e utilizado como instrumento um questionário. Para testar as hipóteses, foi utilizada a estatística não paramétrica Rho de Spearman. Os resultados obtidos de acordo com os testes de hipóteses, para a hipótese geral, o valor does erro calculado entre as variáveis, tem como resultado $(3,4207 \mathrm{E}-63)$ que é menor que a margem $(0,05)$ então pode-se afirmar que há correlação. Em relação ao coeficiente de correlação, obtevese $(0,944379)$, razão pela qual se verifica que a correlação tem sido positiva e muito elevada entre as competências sociais e as dimensões do clima social familiar (relacionamento, desenvolvimento e estabilidade), onde um valor de 0,$870704 ; 0,903853$ e 0,903853, respectivamente

Palavras-chave:

Relacionamento intrafamiliar; Processos de aprendizagem social e apoio familiar 


\section{INTRODUCCIÓN}

Es importante resaltar la el rol que tiene la familia en la vida de todo individuo, es ahí donde se forjan los valores y dinámicas que serán usadas a lo largo de su vida, además es el primer y más importante círculo social que tiene, es por eso que la comunidad de investigadores resalta la importancia de un buen clima familiar para que los estudiantes tengan un buen desempeño social y educativo.

La familia es una construcción teórica de actitudes, rasgos y comportamientos de los integrantes, estos están en equilibrio y son satisfactorios de acuerdo a que tan integrado están los miembros a los valores y actitudes que estimulan el buen clima familiar; por lo que un adecuado clima familiar favorece a la familia en general, sumando conductas positivas y creencias a la dinámica familiar permitiendo entender su constitución, estructura y operatividad (Beneyto, 2015).

Los primeros conocimientos e interacciones se fomentan en la familia, siendo esta la primera referencia que tenemos. Es aquí donde se establecen los roles que seguimos en la sociedad. Se considera a la familia, como el vínculo de personas que establecen relaciones recíprocas e independientes dentro de este grupo social (Mazo, et al., 2019).

En los últimos años muchos de los integrantes de las familias se han visto afectados por la ruptura en su estructura y por otros factores, al respecto, el Fondo de Las Naciones Unidas para la Infancia - Unicef (2016) señala que dentro de poco mucho de los adolescentes tendrán diversos problemas relacionados con la violencia intra familiar, ya que al menos 3/4 partes de la población infantil reciben castigo psicológico y/o físico. Esta problemática es alarmante, ya que impacta en diferentes aspectos de la vida del infante, considerando que el clima familiar influye significativamente en el desenvolvimiento social, afectivo, intelectual y físico de sus integrantes (Moreno, et al., 2009).

En razón al rendimiento académico, muchas investigaciones concluyen que la familia es un factor determinante en los procesos académicos, siendo el acompañamiento y dedicación de la familia no solo beneficiosos en el aspecto académico, sino que brinda herramientas sociales, formando individuos emocionalmente sanos, con capacidades, disciplina y hábitos saludables. (Lastres, López, y Alcázar, 2017). Las habilidades sociales desarrolladas en estudiantes, trae consigo la disminución de probabilidad de conflictividad en el aula, violencia, agresividad entre pares, entre otros. (Estrada, Uchasara, y Gallegos, 2020).

En el ámbito local, con respecto al desenvolvimiento de estas habilidades en estudiantes de secundaria, se ha venido desarrollando en instituciones educativas de la UGEL No 05 ubicadas en SJL, esto a través del trabajo con sus familiares, de manera que los alumnos puedan relacionarse de manera asertiva en su círculo social y familiar

Bajo estos considerados se formuló el siguiente problema general ¿Cuál es la relación entre el clima social familiar y las habilidades sociales en estudiantes de las instituciones educativas UGEL No 05, ¿San Juan de Lurigancho, Perú? Por otro lado, los problemas específicos comprendieron la determinación de la relación que existe entre las dimensiones desarrollo, relación yestabilidad con las habilidades sociales en estudiantes pertenecientes a las instituciones educativas UGEL No 05 , ubicadas en San Juan de Lurigancho, Perú.

El objetivo general del estudio fue establecer la relación entre el clima social familiar y las habilidades sociales, por otro lado, los objetivos 
específicos determinaron la relación entre las dimensiones relación, desarrollo y estabilidad con las habilidades sociales, todos los objetivos mencionados se evaluaron en escolares de las instituciones educativas UGEL No 05 ubicadas en el distrito de San Juan de Lurigancho, Perú.

La hipótesis general formulada para la investigación, plantea que las variables clima social familiar y habilidades sociales se relacionan significativamente. Por otro lado, las hipótesis especificas plantean que las dimensiones relación, desarrollo y estabilidad tienen relación con las habilidades sociales.

El presente estudio, desde una perspectiva teórica, brinda una revisión actualizada y especializada sobre la relación entre las variables clima social familiar y las habilidades sociales en estudiantes, abarcando publicaciones nacionales e internacionales, incrementando los conocimientos relacionados a las variables de estudio y sus dimensiones; es relevante socialmente, ya que la temática y el grupo etéreo que describe pertenece a niños en edad escolar. Su justificación práctica se fundamenta en que el estudio brinda alternativas de solución y brinda resultados útiles para las instituciones educativas, lo que permitirá la toma de decisiones. En su valor metodológico, el estudio utiliza instrumentos estandarizados que permiten establecer mediciones. En su valor social, la investigación aporta un diagnóstico de las escuelas de San Juan de Lurigancho, Perú, lo que permite conocer a profundidad la realidad problemática del lugar así poder tomar decisiones que permitan al alumno desenvolverse en el aspecto educativo y social.

\section{Revisión teórica}

Lastre et al. (2017) determinaron la relación entre el apoyo familiar y el rendimiento académico de estudiantes del $3^{\circ}$ grado de primaria de la IE Heriberto García, Sucre, Colombia. El estudio fue correlacional y tuvo una muestra de 98 estudiantes y 92 familias, se utilizó el análisis de correspondencia múltiple y estadística descriptiva. El resultado de la investigación demostró la correlación entre el rendimiento académico las variables apoyo familiar y rendimiento académico en los cursos de ciencias naturales, lengua castellana matemáticas y ciencias sociales. Llegaron a la conclusión que existen determinantes que permiten lograr el buen rendimiento en el estudiante como el tiempo que le dedican los padres a estudiar, aconsejas y ayudar a responder a las exigencias académicas. Obteniendo más rendimiento los hijos cuyos padres les brindan un acompañamiento escolar.

Rodríguez y Guzmán (2019) determinaron la importancia de los factores de riesgo socio familiares en el rendimiento académico y resaltaron como las variables individuales de los estudiantes intervienen a fin de evitar una repercusión negativa en el ámbito académico. Para realizar este estudio profundizaron en la bibliografía, analizando la relación existente entre el nivel socioeconómico y otras variables de naturaleza familiar. La investigación concluye que el nivel socioeconómico ha sido uno de los factores de riesgo más estudiado y de la bibliografía se puede concluir que el nivel socioeconómico tiene repercusión en el rendimiento del estudiante y se conecta con otros factores de riesgo. 
Pairumani Ticona (2017) en su estudio, determinó la influencia de las relaciones intrafamiliares y el rendimiento académico en alumnos de la Comunidad de Educación Integral, Bolivia. La población fue de 39 estudiantes, tomando como muestra el total. Siendo que 20 alumnos tienen 10 años y 19 alumnos 11 años. El estudio fue de tipo correlacional y de diseño no experimental. Se utilizó como instrumento la escala de Evaluación de Relaciones Intrafamiliares. Se concluye que el rendimiento académico no se ve influido en gran escala a pesar de que las familias que atraviesen por problemas. Por otro lado, las niñas de edades entre los 10 y 11 años obtuvieron mayor promedio y desempeño académico, en comparación con los niños. Por lo tanto, se puede afirmar que, para este estudio, la variable madurez y género, repercutieron directamente en los promedios.

Ramírez (2017), en su investigación realizada en Cajamarca-Perú, determinó la relación entre las variables clima social familiar y habilidades sociales. La muestra fue de 75 estudiantes de primaria en edades de 10 a 12 años. Se utilizaron como instrumentos cuestionarios relativos al clima social familiar y al desarrollo de las habilidades de interacción en niños. Los resultados del estudio mostraron una significativa relación entre las variables, con respecto a la dimensión relaciones y dimensión desarrollo, estas fueron las dimensiones predominantes. En el caso de las habilidades sociales, las habilidades predominantes fueron las habilidades de interacción social y las que se relacionan con las emociones y sentimientos. Concluyendo que las habilidades sociales se fortalecen con un buen clima social familiar.

Gago et al. (2020), en su investigación realizada en la UNMSM, Lima, Perú; determinaron la relación entre el clima social familiar y el rendimiento académico de los estudiantes de la facultad. El tipo de investigación fue básica correlacional, el diseño fue descriptivo correlacional. Los métodos utilizados fueron, el método científico, el hipotético descriptivo y el descriptivo. La población del estudio fue de 1525 estudiantes y la muestra probabilística de 245 estudiantes. Los instrumentos utilizados fueron el test de Moss para la variable clima social familiar, cuya confiabilidad fue comprobada mediante el Alfa de Cronbach dando como resultado 0,954; para la variable rendimiento académico se consideró el historial académico. Los resultados mostraron una significancia $5 \%$ de correlación entre las variables, siendo esta correlación altamente significativa, por lo que se concluye que, a mejor clima familiar, habrá un mejor rendimiento académico.

Por su parte, Carrasco et al. (2020) se propusieron como objetivo determinar la relación entre depresión satisfacción familiar y rendimiento académico en adolescentes de una I.E de Huánuco. El estudio fue de tipo no experimental con diseño transeccional-correlacional; la población de la investigación fue de 593 alumnos y la muestra probabilista fue de 234 adolescentes de 4to y 5to grado de secundaria. Se utilizaron como instrumentos una escala de satisfacción familiar, una adaptación del inventario de Depresión de Beck-II y el historial académico de los alumnos. Para el análisis de los datos utilizaron el estadístico SPSS. Como resultado a través de la prueba de Chi cuadrado se obtuvo $0.0 \%$ de probabilidad, por lo que concluyen que existe relación entre las variables depresión, satisfacción familiar y rendimiento académico en la muestra estudiada.

Acerca de la variable clima social familiar a continuación se definirán los conceptos relacionados:

García (2005) relaciona el clima social familiar con las interacciones de padres e hijos en el hogar, esto varía en cada familia en cantidad y calidad. Las relaciones sociales que establecen los individuos a temprana edad, influye en diferentes etapas de 
su vida, lo que puede beneficiar o no las distintas actividad formativas, educativas, sociales $\mathrm{y} / \mathrm{o}$ familiares. Por esto, es importante la interacción de los padres e hijos, ya que influye directamente en las diversas esferas de la vida humana.

Vargas (2009) define el clima familiar como el lugar donde se forma y se genera la personalidad del púber, por lo que es esencial para el desarrollo de su autoestima, hábitos, disciplina y responsabilidad. Por otro lado, Moos (1984) destaca la importancia del ambiente de familia para la formación de la conducta humana, influyendo a lo largo del crecimiento de la persona.

Zaldívar (2013) define el concepto funcionamiento familiar como las interacciones $\mathrm{y}$ vínculos exitosos entre los miembros, lo cual permite el fortalecimiento de los valores éticos, emocionales, culturales y espirituales.

Goldstein (1980) precisa las habilidades sociales como el conjunto de comportamientos interpersonales que hacen posible las interrelaciones sociales positivas. Por lo que, las habilidades sociales permiten al individuo relacionarse de una manera asertiva y satisfactoria con la sociedad.

Las dimensiones deben ser evaluadas desde los diferentes tipos de interacciones y relaciones interpersonales que se desarrollan al interior de las familias. Según Vargas (2009), se debe considerar las dimensiones relación, desarrollo y estabilidad.

Con respecto a las relaciones familiares, estas están compuestas por las permanentes interacciones diario entre los miembros de la familia, el tipo de relación y comunicación dentro del ámbito familiar marca su estilo de vida; a través del tiempo se va formando una red interactiva entre los miembros, esta es diferente en cada grupo familiar. (Fairlie y Frisancho, 1998) En la familia se dan variadas relaciones, esto los hijos lo ven y perciben diariamente, influyendo en ello positiva o negativamente (García, 2005).
El desarrollo familiar, está conformada por la dinámica del grupo familiar, la cual determina el modo de vida de la familia. (Restrepo, 1993). En este sentido en la familia es común que se tomen decisiones acerca de las actividades cotidianas como, por ejemplo, actividades recreativas, religiosas, sociales, culturales entre otras. (García, 2005).

Con respecto a la estabilidad familiar, esta dimensión se trata del equilibrio y solidez de la familia en los aspectos económicos, emocionales y físicos, por lo que aquí la labor de los padres es primordial para garantizar armonía y solidez. Algunos de los indicadores de estabilidad familiar son la planificación de las actividades en general, el cumplimiento de las normas de convivencia, el cuidado de la salud, establecer tiempos de ocio, entre otros (García, 2005).

Con respecto a la variable habilidades sociales, a continuación, se presentan algunos conceptos:

Según Caballo (2005) las habilidades sociales comprenden el conjunto de conductas con las que el individuo puede desarrollarse individual o interpersonalmente, expresando sus deseos, opiniones, puntos de vides, entre otros, de manera adecuada a las circunstancias. Por lo general estas habilidades le permiten resolver y disminuir problemas en su día a día en la medida que el individuo respeta las conductas de los otros. (León Rubio y Medina Anzano ,1998).

Bandura (1987) señala que las habilidades sociales contienen dos fases importantes, la primera se refiere a los procesos de mediación que se refieren a los estímulos y respuestas, la segunda fase es la conducta aprendida del medio a través de la observación. Es por esto que la teoría del aprendizaje social es llamada aprendizaje por observación.

El autor antes mencionado expresa que existen cuatro procesos para el aprendizaje social; primero, la atención la cual debe estar focalizada para no obstaculizar el aprendizaje con distractores; 
segundo, la retención, el comportamiento adquirido debe ser almacenando en la memoria; tercero, la reproducción, el individuo debe tener la capacidad de reproducir la conducta adquirida; finalmente, la motivación, se tiene que querer realizar la conducta adquirida.

Dongil y Cano (2015) señalan que un individuo con habilidades sociales tiene que tener la capacidad de presentarse y conducirse ante otras personas, permitiendo al individuo establecer relaciones positivas en su ambiente social.

\section{MÉTODO}

Se empleó el enfoque cuantitativo, según Hernández y Mendoza (2018), este realiza la medición de las variables de estudio, en esta investigación, clima social familiar y las habilidades sociales. Por otro lado, el tipo de investigación es investigación básica, ya que recopila información con el objetivo de producir conocimiento y teorías, (Sánchez y Reyes, 2017). El diseño fue no experimental, transversal-correlacional, ya que según (Ary et al.,1989) un estudio correlacional pretende determinar la relación o grado entre dos variables. Permitiendo evaluar hasta qué punto las alteraciones de una variable alteraran la otra variable.

La población del estudio fue de 243 estudiantes pertenecientes al 3er grado de secundaria de las instituciones educativas de la UGEL No 05 , ubicadas en el distrito de San Juan de Lurigancho. Aplicando la fórmula del tamaño de la muestra se obtuvo 129 estudiantes.

Se utilizó como instrumento un cuestionario que evaluó las dos variables estudiadas, que consistió en preguntas relacionadas a las variables que serán medidas (Hernández, Fernández y Baptista, 2014). La validez de los instrumentos se fundamenta en el Alarcón (1991) que desarrolló la lista de chequeo de Goldstein. Para la confiabilidad de los instrumentos estos se sometieron al estadístico Alfa de Cronbach, donde los instrumentos presentaron alta confiabilidad.

\section{RESULTADOS Y DISCUSIÓN}

\section{Sobre los resultados inferenciales}

Para la hipótesis general, el valor de error calculado entre las variables, el resultado $(3,4207 \mathrm{E}$ 63 ) es inferior al margen $(0,05)$ por lo que se puede afirmar que existe correlación. Con respecto al coeficiente de correlación se obtuvo $(0,944379)$ por lo que se comprueba que la correlación ha sido positiva y muy alta. En consecuencia, se acepta la hipótesis alterna: Existe una relación significativa entre el clima social familiar y las habilidades sociales en estudiantes de las instituciones educativas UGEL No 05, San Juan de Lurigancho, Perú. (Tabla 1).

Con respecto a la hipótesis específica 1 , en la dimensión relación y la variable habilidades sociales se obtuvo como valor de error calculado un resultado de 5,8371E-41 y el coeficiente de correlación 0,870704 ; para la hipótesis específica 2 para la relación entre la dimensión desarrollo y habilidades sociales el resultado de error fue de $1,1625 \mathrm{E}-48$ y el coeficiente de correlación calculado 0,903853; para la hipótesis especifica 3 , el valor de error entre la dimensión desarrollo y la variable habilidades sociales dio como resultado 1,1625E-48 y el coeficiente de correlación calculado 0,903853 .

Para las tres hipótesis específicas, el resultado del valor de error calculado es inferior a lo establecido $(0,05)$, por lo que se afirma que existen una correlación. Para el caso del coeficiente de correlación, según los resultados en las tres hipótesis específicas, se comprueba que la correlación es muy alta y positiva. (Tabla 1). 
Tabla 1. Resultados de la prueba de correlación de Spearman para la hipótesis general y específicas.

\begin{tabular}{lllc}
\hline & & & $\begin{array}{c}\text { Variable 2 } \\
\text { Habilidades sociales }\end{array}$ \\
\hline Hipótesis General & $\begin{array}{l}\text { Variable 1 } \\
\text { Clima social familiar }\end{array}$ & Coeficiente de correlación & 0,944379 \\
& Error calculado & $3,4207 \mathrm{E}-63$ \\
Hipótesis Específica & $\begin{array}{l}\text { Dimensión 1 } \\
\text { Relación }\end{array}$ & Coeficiente de correlación & 0,870704 \\
Hipótesis Específica 2 & $\begin{array}{l}\text { Dimensión 2 } \\
\text { Desarrollo }\end{array}$ & Error calculado & $5,8371 \mathrm{E}-41$ \\
& Coeficiente de correlación & 0,903853 \\
Hipótesis Específica 3 & $\begin{array}{l}\text { Dimensión 3 } \\
\text { Estabilidad }\end{array}$ & Error calculado & $1,1625 \mathrm{E}-48$ \\
& Coeficiente de correlación & 0,857634 \\
\hline
\end{tabular}

\section{Discusión}

Con respecto a los resultados del estudio, se evidenció una correlación muy alta entre las variables clima social familiar y habilidades sociales, por lo que tener un buen clima familiar fortalece las habilidades sociales de los estudiantes, razón por la cual coincidimos con los aportes de Ramírez (2017), que en su estudio realizado en Cajamarca-Perú, concluyen también que las variables habilidades sociales y clima social familiar se correlacionan significativamente. Por otro lado, las dimensiones predominantes obtenidas por el autor fueron las dimensiones de relación y desarrollo; Estos resultados nos permiten comparar las hipótesis específicas 1 y 2, que relacionan estas dimensiones con las habilidades sociales.

El estudio de Gago et al. (2020), sobre la relación entre el clima social familiar y el rendimiento académico de los universitarios de la facultad de educación de la Universidad Mayor de San Marcos; permitió la revisión de la literatura y observar la metodología e instrumentos utilizados. Además, coinciden en la conclusión del estudio ya que los resultados de la investigación mostraron una significancia $5 \%$ de correlación entre las variables, siendo esta correlación altamente significativa, por lo que concluyen que, a mejor clima familiar, habrá un mejor rendimiento académico.

Los resultados obtenidos contrastan con Pairumani Ticona (2017), quien, en su estudio realizado en Bolivia, para una muestra 39 estudiantes con edades entre los 10 y 11 años, evidencia que los problemas por los que atravesaban las familias no influyeron sustancialmente en el rendimiento académico. Por otro lado, resalta la importancia del desarrollo humano y las diferencias individuales

Lastre et al. (2017) realizaron un estudio en el que determinaron la relación entre el apoyo familiar y el rendimiento académico de estudiantes del $3^{\circ}$ grado de primaria concluyendo que cuando los hijos son acompañados académicamente por los padres, muestran mejor desempeño académico. Estos resultados nos permiten comparar la influencia de la familia y el clima familiar en diferentes áreas.

CONCLUSIONES

Se afina que existe una relación entre el clima social familiar y las habilidades sociales y esta 
correlación es altamente significativa, por lo que un buen clima social familiar mejora las habilidades sociales de los estudiantes de las instituciones educativas UGEL No05-San Juan de Lurigancho, Perú.

Con respecto a las hipótesis específicas que midieronla relación entrelas dimensiones relaciones, desarrollo y estabilidad con las habilidades sociales, según el coeficiente de correlación obtenido en cada caso se concluye que existe una correlación positiva $\mathrm{y}$ altamente significativa.

\section{REFERENCIAS}

Alarcón, R. (1991). Métodos y diseños de investigación del comportamiento. Lima: Universidad Peruana Cayetano Heredia [2da. edición, ampliada, Lima, Universidad Ricardo Palma, 2008)]

Ary, D. Jacobs, L y Razavieh, A. (1989). Introducción a la Investigación Pedagógica. Segunda edición. México. Mcgraw-Hill

Bandura, A. (1987) Pensamiento y Acción. Barcelona: Martínez Roca

Beneyto, S. (2015). Entorno familiar y rendimiento académico. España: Área de innovación y desarrollo

Caballo, V. (2005). Manual de Evaluación y entrenamiento de las habilidades sociales. $\left(6^{\circ}\right.$ Edición). Madrid: Siglo XXI

Carrasco-Muñoz, M. A.., Martínez-Morales, C., Noreña-Tello, F., y Bao-Condor, C. L. (2020). Satisfacción familiar, depresión y rendimiento académico en adolescentes de un Colegio Estatal de Huánuco, Perú. Revista Boletín Redipe, 9(2), 197-210. https://doi.org/10.36260/rbr.v9i2.922

Dongil, E., y Cano, A. (2015) Habilidades sociales. España: SEAS.

Estrada Araoz, E., Mamani Uchasara, H., y Gallegos Ramos, N. (2020). Estrategias psicoeducativas para el desarrollo de las habilidades sociales de los estudiantes de educación secundaria. Revista San Gregorio, (39), 116-129. https://doi. org/6http://doi.org/10.36097/rsan.v1i39.1374
García, C. (2005). Habilidades Sociales, Clima Social Familiar y Rendimiento Académico en Estudiantes Universitarios. Universidad de San Martín de Porres, Escuela Profesional de Psicología. ISSN: 1729 - 4827. Lima-Perú

Golstein, A. (1980). Competencia social: el cambio del comportamiento individual en la comunidad. México: Trillas

Hernández-Sampieri, R., y Mendoza, C. (2018). Metodología de la investigación. Las rutas cuantitativa, cualitativa y mixta. México. McGrawHill.

Hernández, R., Fernández, C. y Baptista, P. (2014). Metodología de la investigación científica. México D. F: Mc Graw-Hill

Lastre Meza, K., López Salazar, L. D., y Alcázar Berrio, C. (2017). Relación entre apoyo familiar y el rendimiento académico en estudiantes colombianos de educación primaria. PSICOGENTE, 21(39). https://doi. org/10.17081/psico.21.39.2825

León Rubio, J. y Medina Anzano, S. (1998). Aproximación conceptual a las habilidades socailes. En F. Gil y J. León (Edit.). Habilidades sociales. Teoría, investigación e intervención (pp. 13-23). Madrid: Síntesis Psicología

Mazo, Y. I., Mejía, L. A., y Muñoz, Y. P. (2019). Calidad de vida: la familia como una posibilidad transformadora. Revista Poiésis, 36(1), 98-110

Moos, R., Moos B., y Trickett, E. (1984). FES, WES y CES. Escalas de clima social. TEA Ediciones

Moreno, D., Estévez, E., Murgui, S., y Musitu, G. (2009). Relación entre el clima familiar y el clima escolar: el rol de la empatía, la actitud hacia la autoridad y la con- ducta violenta en la adolescencia. International Jour- nal of Psychology and Psychological Therapy, 9(1), 123- 136

Gago, D., Ruiz Tejada, J. O., Hurtado Tiza, D. R., y Añaños Bedriñana, M. A. (2020). Clima social familiar y ren- dimiento académico en estudiantes de una universidad pública de Lima. Revista Conrado, 16(77), 26-31 
Ramírez, Y. (2017). Clima social familiar y habilidades sociales en estudiantes de educación primaria de Cajamarca. Revista Paian, 8(2), 6370

Pairumani Ticona R. (2017). Influencia de las relaciones intrafamiliares, en el rendimiento académico en niños (as) de 10 y 11 años, que asisten a la Comunidad de Educación Integral (CEI). Revista de Investigación Psicológica, (2016), 87-108. Retrieved from http:// www.scielo.org.bo/scielo.php?pid=S2223$0322017000200008 \& \mathrm{script}=\mathrm{sci}$ abstract\&tlng $=\mathrm{pt}$
Sánchez, H y Reyes, C. (2014). Metodología y diseños en la investigación científica. Perú: Visión universitaria

Vargas, J. (2009). Percepción de clima social familiar $\mathrm{y}$ actitudes ante situaciones de agravo en la adolescencia tardía. Revista Interdisciplinaria. Argentina: Redaly. Org 